Document downloaded from:

http://hdl.handle.net/10251/84436

This paper must be cited as:

Torregrosa, AJ.; Broatch, A.; Gil, A.; Moreno Martinez, D. (2012). Analysis of acoustic networks including cavities by means of a linear finite volume method. Journal of Sound and Vibration. 331(20):4575-4586. doi:10.1016/j.jsv.2012.05.023.

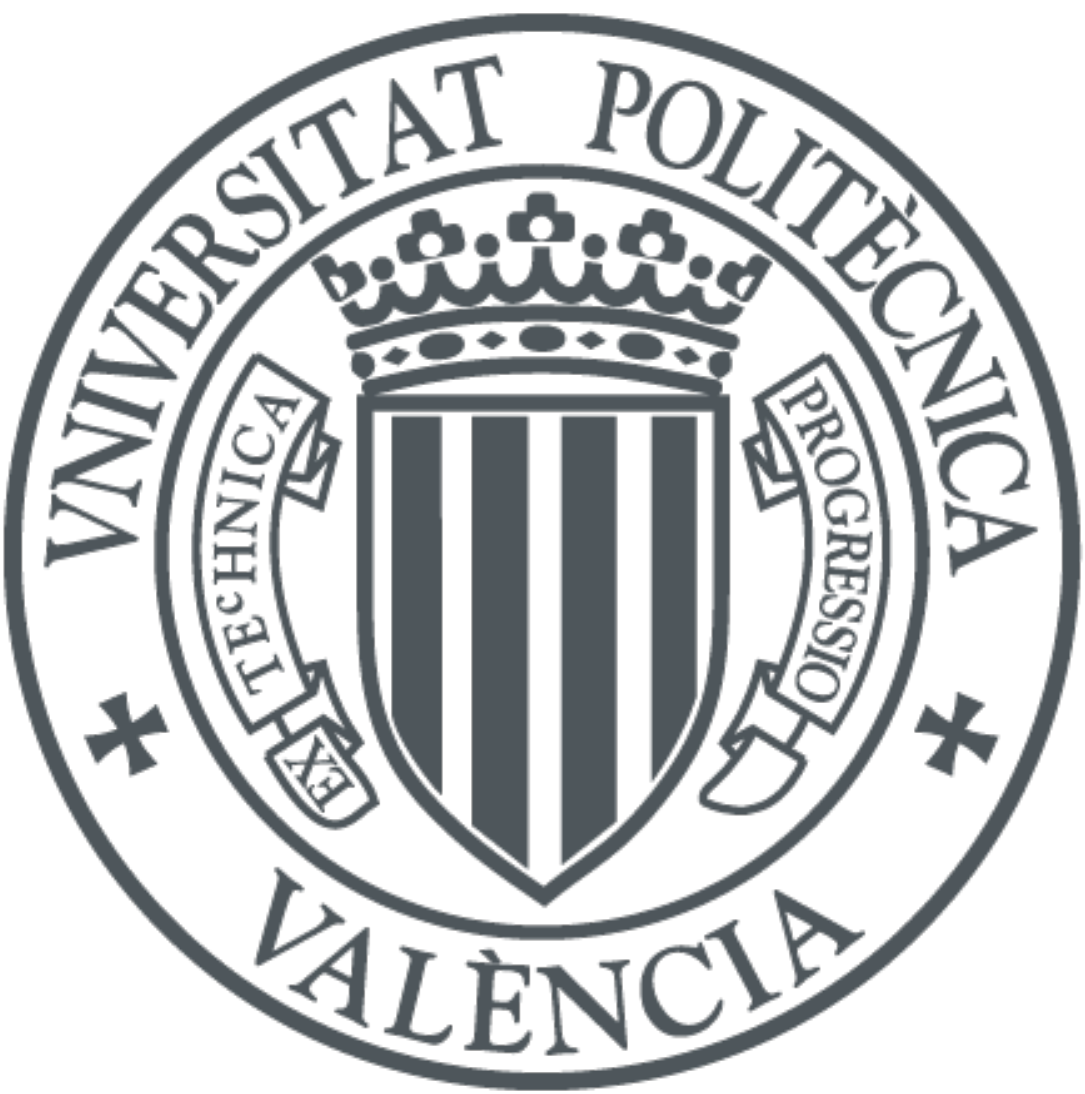

The final publication is available at

http://dx.doi.org/10.1016/j.jsv.2012.05.023

Copyright Elsevier

Additional Information 


\title{
Analysis of acoustic networks including cavities by means of a linear finite volume method
}

\author{
A.J. Torregrosa*, A. Broatch, A. Gil, D. Moreno \\ CMT-Motores Térmicos, Universitat Politècnica de València, Aptdo. 22012, E-46071 Valencia, Spain.
}

\begin{abstract}
A procedure allowing for the analysis of complex acoustic networks, including three-dimensional cavities described in terms of zero-dimensional equivalent elements, is presented and validated. The procedure is based on the linearization of the finite volume method often used in gas-dynamics, which is translated into an acoustic network comprising multi-ports accounting for mass exchanges between the finite volumes, and equivalent 2-ports describing momentum exchange across the volume surfaces. The application of the concept to a one-dimensional case shows that it actually converges to the exact analytical solution when a sufficiently large number of volumes are considered. This has allowed the formulation of an objective criterion for the choice of a mesh providing results with a prefixed error up to a certain Helmholtz number, which has been generalized to three-dimensional cases. The procedure is then applied to simple but relevant three-dimensional geometries in the absence of a mean flow, showing good agreement with experimental and other computational results.
\end{abstract}

Keywords: Acoustic network, Finite volume, Acoustical cavities

\section{Introduction}

The parametric study of possible solutions plays an important role in the design process of acoustic systems, including engine intake and exhaust systems. For this purpose, models based on one-dimensional methods are those mostly used, due to their extremely low computational cost, even when relatively complex acoustic networks are considered.

The determination of the acoustic behaviour of an arbitrarily complex network consisting of one dimensional elements was first addressed by Eversman [1], who proposed a systematic procedure for the analysis of multiply branched acoustic transmission lines using a scheme similar to the assembly methods of finite element analysis. Subsequent efforts were reported by Glav and Åbom [2], who developed a general formalism for analyzing acoustic 2-port networks in which the junctions between 2-ports may be considered as active multi-ports. Dowling and Peat [3] described an efficient algorithm based on the identification of subsystems of 2-port acoustic elements. Later, Panigrahi and Munjal [4] proposed an algorithm for studying the acoustic behaviour of a system of interconnected rigid walled acoustic filter elements that makes use of a connectivity matrix in order to automate the generation of pressure balance equations and volume-velocity continuity equations at the junctions, along with the proper transfer matrices between them.

${ }^{*}$ Corresponding author. Tel.: +3496 3877650, fax: +34 963877659 .

Email address: atorreg@mot.upv.es (A.J. Torregrosa) 
All these methods provide an efficient solution of the overall problem inasmuch as the basic elements may be represented by a 2-port. Of course, consideration of elements exhibiting three-dimensional effects requires, in principle, that the corresponding 2-port is imported from a different calculation.

The standard tools for the three-dimensional analysis of acoustic cavities are finite element methods (FEM) $[5,6]$ and boundary element methods (BEM) $[6,7,8]$, due to their adaptability to any type of geometry. However, these methods require a high computational cost and thus, in spite of the good results obtained, their use may not be advisable in acoustic networks in which most of the elements admit a onedimensional description. In this sense, by using analytical methods the computation time may be reduced without penalty in the accuracy of the results. A wide variety of papers study the analytical solution for the case of expansion chambers of rectangular $[9,10]$, circular $[11,12,13]$ and elliptical [14] cross-section. The major drawbacks of this approach are, on one hand, the complicated algebra involved and, on the other hand, the fact that analytical methods cannot be applied to geometries whose eigenfunctions are not readily known. This problem increases in difficulty when mean flow or absorbent materials [15] are included. Computational Fluid Dynamics (CFD) techniques have also been developed in order to calculate the acoustic response of mufflers, taking into account the nonlinear dissipation associated with the interaction between the flow and the acoustic field [16]. In general, the CFD method is more convenient for complex geometries in the presence of a mean flow, where convective and dissipative effects may be determinant [17]. However, this technique entails a considerable computational effort to obtain a complete and accurate solution, so that the same comment as for FEM and BEM applies to its eventual use for large acoustic networks. This method is particularly useful for the identification of sources of dissipation inside the mufflers, which could later be included into less demanding linear calculations through the use of local impedances, resistive terms, etc.

The inclusion of information obtained with any of these three-dimensional techniques in an otherwise one-dimensional network is relatively straightforward. Eversman and Ricci [18] developed a cavity element especially well suited for inclusion in the procedure described in [1]. However, efforts have been also reported on the possibility to describe three-dimensional effects by means of one-dimensional elements. Of course, this may imply some a priori knowledge on the relevant dimensions of the device under study, as in the works of Torregrosa et al. [19] and Mimani and Munjal [20], where short reversal chambers were studied on the basis of plane wave propagation in the transversal direction. A more general procedure was proposed by Panigrahi and Munjal [21] which is based on the volume synthesis algorithm and allows the characterization of the acoustic response of relatively complex devices by using the transfer matrices with plane-wave approximation.

Recently, Amphlett et al. [22] have proposed a different approach for the inclusion of three-dimensional effects, in which a cavity is represented as a network of zero-dimensional nodes and equivalent 2-ports, so that an arbitrarily complex acoustic network including cavities may be represented solely in terms of 2-ports and nodes. This formulation arises naturally from the linearization of the finite volume approach as used in gas-dynamic simulations [23]. In [22] it was only checked that the results obtained with this approach were in good agreement with those obtained from a gas-dynamic finite-volume time domain simulation, but a detailed justification of the procedure and comparison with experiments and other computational methods were missing. These aspects are dealt with in the present paper.

The paper is organized as follows. First, the methodology proposed in [22] is described for completeness, with especial emphasis on the linear description of the junctions between 2-ports. In section 3, the one-dimensional case of a uniform duct portion without mean flow is considered in detail, showing that the proposed procedure actually converges to the exact analytical solution. As a by-product of this analysis, an objective criterion is obtained for the mesh spacing required in order to produce reliable results up to a given frequency with a certain prefixed error, which is illustrated in the case of the one-dimensional response of a 
circular expansion chamber. Then, in section 4 the performance of the proposed method is checked in two cases which exhibit three-dimensional effects, and comparison with experimental and other computational results in the absence of a mean flow is presented and discussed. Finally, in the last section the conclusions obtained from the present study are summarized.

\section{Theory}

The numerical methodology used in this paper is based on a linear version of finite volume method. In the following, the linearization procedure is described, showing that the linearized mass and momentum equations may be interpreted in terms of volume-related junctions connected by means of length- and arearelated equivalent 2-ports. In the frame of a finite volume time-domain calculation, a given cavity would be regarded as built of smaller volumes, so that within such a description a staggered mesh system is used. Then, once the volumes are defined, equations of mass and energy conservation are solved for each volume, and the momentum equation is solved for each interface between two volumes [24]. The energy equation will not be considered, as the flow evolution inside the volumes will be regarded as isentropic, so that only the mass continuity equation and the momentum equation need to be addressed. First, according to Morel et al. [24], mass conservation in a given fluid volume can be expressed as:

$$
\frac{\mathrm{d} m_{n}}{\mathrm{~d} t}=\sum_{i} \dot{m}_{n}^{(i)}
$$

where $m_{n}$ is the mass contained in the $n$-th volume, and $\dot{m}_{n}^{(i)}$ represents the mass flow corresponding to the $i$-th opening connecting the $n$-th volume to any other volumes. Assuming that the evolution inside the volume is isentropic, one may introduce the pressure $p_{n}$ inside the volume, thus getting

$$
\frac{\mathrm{d} m_{n}}{\mathrm{~d} t}=\frac{V_{n}}{c_{0}^{2}} \frac{\mathrm{d} p_{n}}{\mathrm{~d} t}=\sum_{i} \dot{m}_{n}^{(i)}
$$

Here, $c_{0}$ is the speed of sound and $V_{n}$ is the volume. Now, writing all the variables as the addition of a mean value and a fluctuating part ( $p \rightarrow p_{0}+p$, etc.), and assuming harmonic time-dependence for the fluctuating parts, one finally has

$$
\mathrm{j} \omega \frac{V_{n}}{c_{0}^{2}} p_{n}=\sum_{i} v_{n}^{(i)} \equiv v_{n}
$$

where $\mathrm{j}=\sqrt{-1}, \omega$ is the angular frequency and $v_{n}$ represents the mass velocity fluctuation, i.e. the fluctuating part of the mass flow rate. Therefore, in the pressure-mass velocity representation, the behaviour of the volume regarding mass conservation may be represented by considering a multi-port with a passive impedance given by:

$$
Z^{(n)}=\mathrm{j} \frac{c_{0}^{2}}{\omega V_{n}}
$$

which is the impedance corresponding to the compliance $V / c_{0}^{2}$ of a cavity of volume $V$ [25]. Secondly, according to reference [24], the momentum equation at the interface between volumes can be written as

$$
\frac{\mathrm{d}(m u)}{\mathrm{d} t}+\sum \dot{m} u=-A \Delta p
$$


Here, $u$ is the particle velocity and $A$ is the cross-sectional area at the interface (a loss term in the right-hand side has been omitted), whereas $\Delta p$ represents the pressure difference between the two volumes. Now, if equation (5) is referred to the unit volume one gets

$$
\frac{\mathrm{d}(\rho u)}{\mathrm{d} t}+\sum \frac{\rho u^{2}}{l}=-\frac{\Delta p}{l}
$$

where $l$ is a characteristic length. Following a linearization procedure similar to that used to obtain equation (3), for the case without a mean flow one obtains

$$
\rho_{0} \frac{\mathrm{d} u}{\mathrm{~d} t}=-\frac{\Delta p}{l}
$$

Then, assuming harmonic time dependence for all the fluctuating variables and introducing the mass velocity $v=\rho A u$, gives:

$$
\mathrm{j} \omega \frac{l}{A} v=-\Delta p
$$

It is apparent that a linear relation such as equation (8) admits a simple representation in terms of a 2-port, i.e., a system relating the state variables of two different points. By identifying such points with the volumes themselves, it appears that momentum exchange at the interface between two volumes can be actually represented by a 2-port defined by equation (8), which corresponds to the impedance associated with the lumped inertance $l / A$ of a short duct [25]. In the case that a mean flow is present, equation (3) remains unaffected, whereas equation (8) must be substituted by [22]

$$
\left[\mathrm{j} \omega \frac{l}{A}+2 \frac{U_{0}}{A}\right] v=-\Delta p
$$

where $U_{0}$ is the mean flow velocity across the interface, which can be obtained from a time domain steady flow computation performed with the same spatial discretization, as described by Sapsford et al. [23].

In summary, mass and momentum exchange between volumes may be represented by considering each volume as a multi-port, as defined by equation (3), and then connecting those multi-ports by means of 2 ports as defined by equation (8) or equation (9). In this way, any cavity may be represented by a network of zero-dimensional nodes connected by 2-ports, for which suitable solutions exist in the literature, as commented in the introduction. In the following section, the concept will be first illustrated in the simple case of a one-dimensional uniform duct with no mean flow.

\section{One-dimensional analysis}

Consider the case depicted in Figure 1: a one-dimensional uniform duct is represented as a series of finite volumes of equal length $l$. Then, according to the previous discussion, each of these volumes may be represented as a multi-port connected to the multi-ports representing the adjacent volumes by means of 2-ports. As shown in the detail, multi-ports are assumed to be located at the centre-point of the volume, with two 2-ports of length $l / 2$ on both sides. Actually, in the one-dimensional case considered the multi-ports are simply 2-ports, and all the elements may be represented conveniently by their transfer matrices. Then, the transfer matrix of the whole duct would be obtained making use of the well-known cascading property of transfer matrices.

The transfer matrix of the volume-related 2-ports is, according to equation (3), 


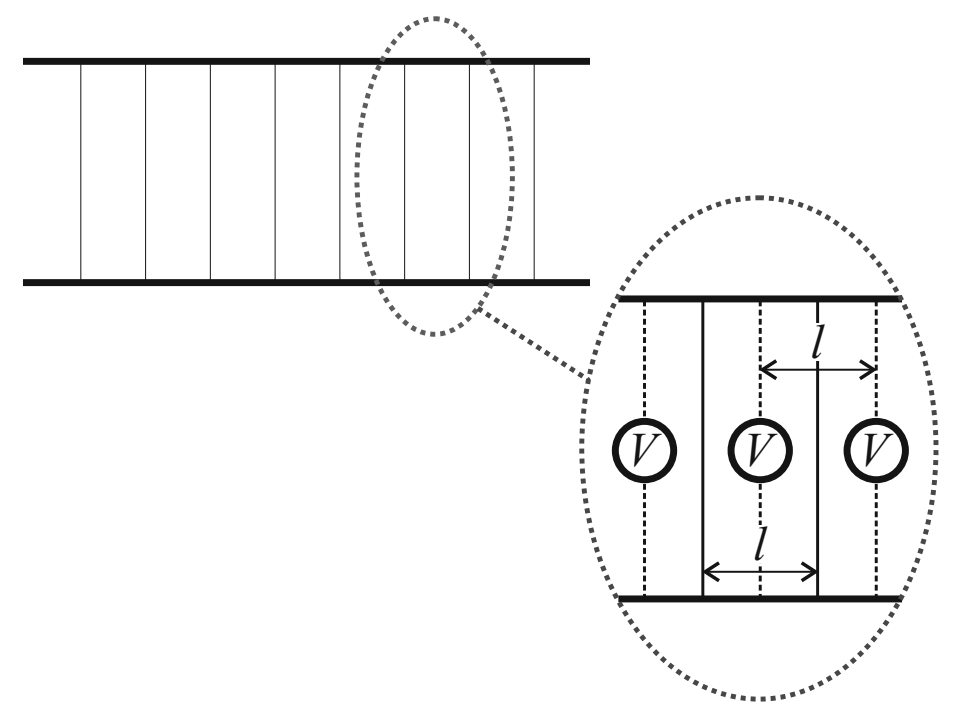

Figure 1: Equivalent representation of one-dimensional finite volumes.

$$
\left[\begin{array}{cc}
1 & 0 \\
\mathrm{j} \omega V / c_{0}^{2} & 1
\end{array}\right]
$$

and that of the momentum-related 2-ports, following equation (8), has the following form:

$$
\left[\begin{array}{cc}
1 & \mathrm{j} \omega l /(2 A) \\
0 & 1
\end{array}\right]
$$

Therefore, the transfer matrix of each volume is obtained by pre- and post-multiplying the matrix in equation (10) by that in equation (11), giving:

$$
\left[\begin{array}{cc}
1-\frac{1}{2}(k l)^{2} & \mathrm{j}\left(c_{0} / A\right)\left[k l-\frac{1}{4}(k l)^{3}\right] \\
\mathrm{j}\left(A / c_{0}\right) k l & 1-\frac{1}{2}(k l)^{2}
\end{array}\right]
$$

Here, $k=\omega / c_{0}$ is the wave number. It is interesting to compare this expression with the exact transfer matrix of a duct portion of length $l$ :

$$
\left[\begin{array}{cc}
\cos k l & \mathrm{j}\left(c_{0} / A\right) \sin k l \\
\mathrm{j}\left(A / c_{0}\right) \sin k l & \cos k l
\end{array}\right]
$$

It is apparent, from equation (12), that the diagonal elements are the two first terms of the Taylor expansion of $\cos k l$. The $k l$ in the left-bottom element can be identified with the first term of the Taylor series for $\sin k l$, and the term in brackets in the top-right element is similar to the first two terms of the Taylor expansion for $\sin k l$. There is thus a clear indication that if the meshing is refined these approximation series will get closer to the exact solution. In order to confirm this, a general expression for a duct of length $L$ divided into $n$ finite volumes of length $L / n$ is obtained in the following. The transfer matrix of such a duct will be given by

$$
\left[\begin{array}{cc}
1-\frac{1}{2}(k L / n)^{2} & \mathrm{j}\left(c_{0} / A\right)\left[k L / n-\frac{1}{4}(k L / n)^{3}\right] \\
\mathrm{j}\left(A / c_{0}\right) k L / n & 1-\frac{1}{2}(k L / n)^{2}
\end{array}\right]^{n} \equiv\left[\begin{array}{ll}
a & b \\
c & d
\end{array}\right]^{n}
$$


As the matrix in equation (12) is diagonalizable, its $n$-th power can be obtained once its eigenvalues and eigenvectors are known. After some algebra one gets:

$$
\begin{aligned}
{\left[\begin{array}{ll}
a & b \\
c & d
\end{array}\right]^{n} } & =\frac{1}{2}\left[\begin{array}{cc}
1 & 1 \\
\sqrt{c / b} & -\sqrt{c / b}
\end{array}\right]\left[\begin{array}{cc}
(a+\sqrt{b c})^{n} & 0 \\
0 & (a-\sqrt{b c})^{n}
\end{array}\right]\left[\begin{array}{cc}
1 & \sqrt{b / c} \\
1 & -\sqrt{b / c}
\end{array}\right] \\
& =\frac{1}{2}\left[\begin{array}{cc}
(a+\sqrt{b c})^{n}+(a-\sqrt{b c})^{n} & \sqrt{b / c}\left[(a+\sqrt{b c})^{n}-(a-\sqrt{b c})^{n}\right] \\
\sqrt{c / b}\left[(a+\sqrt{b c})^{n}-(a-\sqrt{b c})^{n}\right] & (a+\sqrt{b c})^{n}+(a-\sqrt{b c})^{n}
\end{array}\right]
\end{aligned}
$$

Binomial expansion of the different matrix terms gives, after some lengthy calculations,

$$
\begin{aligned}
& T_{11}=\sum_{m=0}^{n}(-1)^{m} \Psi_{m}^{11}(n) \frac{(k L)^{2 m}}{(2 m) !}=T_{22} \\
& T_{12}=\mathrm{j}\left(c_{0} / A\right) \sum_{m=0}^{n}(-1)^{m} \Psi_{m}^{12}(n) \frac{(k L)^{2 m+1}}{(2 m+1) !} \\
& T_{21}=\mathrm{j}\left(A / c_{0}\right) \sum_{m=0}^{n-1}(-1)^{m} \Psi_{m}^{21}(n) \frac{(k L)^{2 m+1}}{(2 m+1) !}
\end{aligned}
$$

where the $\Psi_{m}(n)$ terms are given by:

$$
\begin{aligned}
& \Psi_{m}^{11}(n)=\frac{1}{n^{2 m-1}} \frac{(m+n-1) !}{(n-m) !} \\
& \Psi_{m}^{12}(n)=\frac{n^{2}+m / 2}{n^{2 m+1}} \frac{(m+n-1) !}{(n-m) !} \\
& \Psi_{m}^{21}(n)=\frac{1}{n^{2 m+1}} \frac{(m+n) !}{(n-m-1) !}
\end{aligned}
$$

From equations (16) and (17) it is apparent that, for sufficiently large $n$, the solution obtained converges to the exact solution given by equation (13), as all the $\Psi_{m}(n)$ tend to unity and thus the Taylor expansion is actually approached.

The term that takes longer to converge to the exact solution is $T_{21}$ as it is approximated by the lowest order series. Next, this term is analyzed in depth in order to assess the accuracy of these approximation series. As the transfer matrix of a duct is given by equation (13), the overall error of the solution provided by this approach can be calculated by means of the $L^{2}$-norm. This error, expressed as a function of the value of the Helmholtz number $k L$ required and the number of divisions $n$ considered, is shown in Figure 2.

Next, an example where solutions with different degrees of accuracy are required up to a value of $k L$ equal to 11 will be discussed. From Figure 2, if a horizontal line is drawn at the level of the required value of $k L$, different division numbers are obtained depending on the desired accuracy. For instance, in order to obtain results with an overall error of $5 \%, 1 \%$ and $0.1 \%$, the numbers of divisions required are 11,16 and 28 , respectively. The results obtained when these numbers of divisions are used have been depicted in Figure 3, which shows how the solutions converge towards the exact solution when the number of divisions increases, as expected. Here, it can be observed that 16 divisions provide a sufficiently good concordance with the exact solution. The rest of terms of the transfer matrix have been also analyzed and they converge 


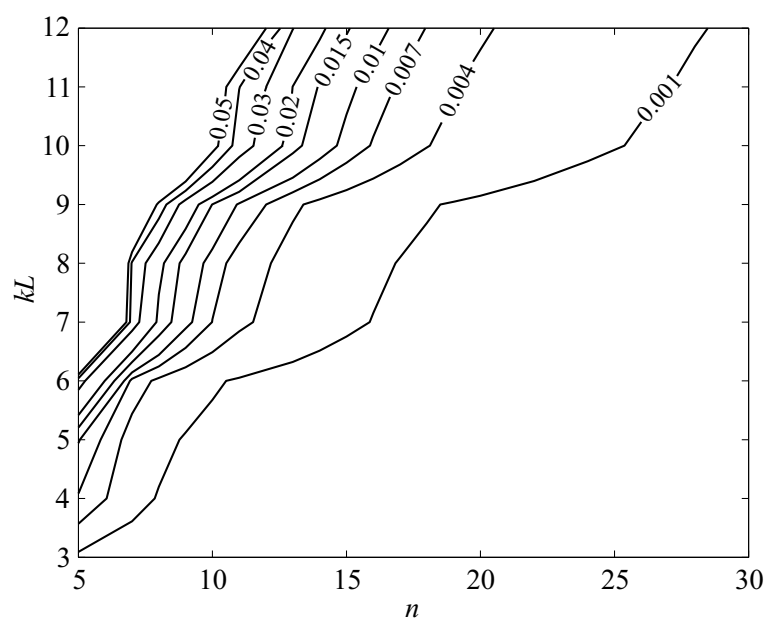

Figure 2: Overall error as a function of the required $k L$ and the number of divisions considered.

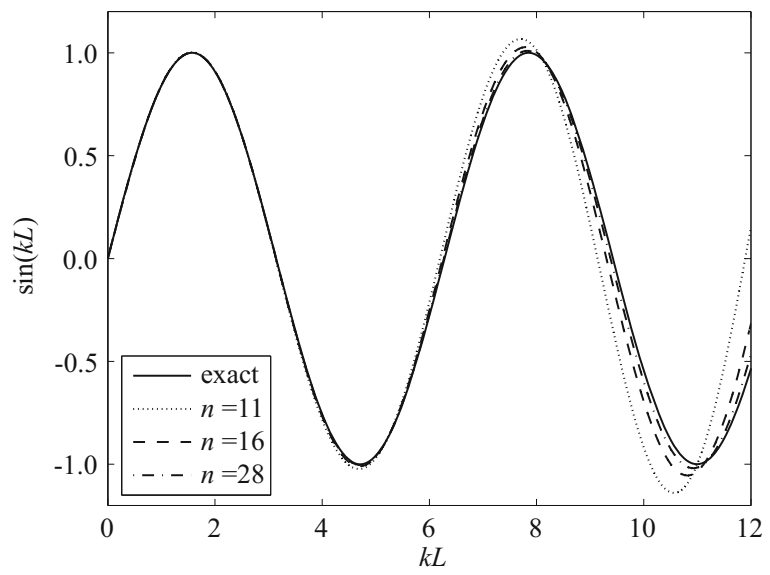

Figure 3: Comparison between the exact expression of $\sin (k L)$ and several approximations obtained with the proposed methodology. 


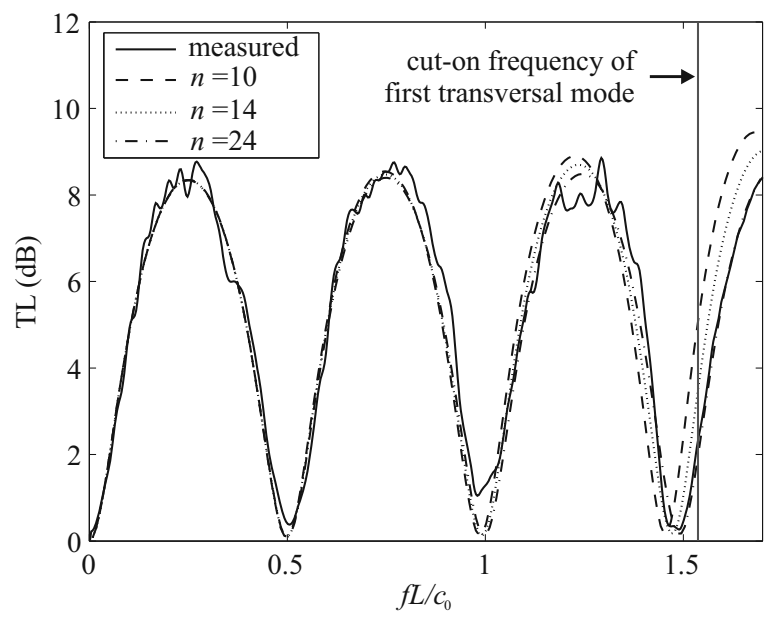

Figure 4: Transmission loss of a circular expansion chamber: comparison of measurements and calculations with different numbers $n$ of divisions.

to the exact solution faster than the term $T_{21}$. Therefore, reliable results can be obtained by considering a relatively low number of divisions.

Further evidence on the suitability of the previous procedure in one-dimensional cases is provided by the analysis of the acoustic response of a circular expansion chamber. In the example considered, the area expansion ratio was set to 5, the chamber length was $300 \mathrm{~mm}$ and the chamber diameter was $114 \mathrm{~mm}$. As a one-dimensional analysis is valid only for frequencies below the cut-on frequency of the first transversal mode, the maximum value of $k L$ to be considered is 9.68. From Figure 2 it is obtained that solutions with an overall error of $5 \%, 1 \%$ y $0.1 \%$ require numbers of divisions of 10,14 and 24 , respectively.

Comparison of the transmission loss provided by the proposed methodology and experimental data is shown in Figure 4 as a function of $f L / c_{0}$. As in the previous analysis, the solution converges towards the experimental data when the number of divisions increase and the results obtained for the value of $n$ that provides an overall error of $1 \%$ show very good agreement with the experimental results. It can thus be concluded that this methodology is able to reproduce the one-dimensional acoustic response of mufflers with a relatively modest discretization. The interest of this result does not lie, however, in its application to one-dimensional systems, where an exact analytic solution is available, but on the possibility of its extension to three-dimensional cases, which is considered next.

In order to extrapolate the result obtained in the one-dimensional analysis to the three-dimensional case, a non-dimensional nodal spacing expression [26] is used, which can be written as [27]:

$$
\frac{L}{n} \leq \frac{c_{0}}{r f}
$$

where $f$ is the frequency up to which accurate results are required, $L$ is the dimension of the cavity in a given direction and $r$ is the number of computational cells per wavelength. From equation (18), a simple relation between the value of $k L$ and the number of divisions, keeping constant the number of computational cells per wavelength, can be obtained. Figure 5 shows a new error map where the optimum value of $r$, according to the number of divisions and the value of $k L$, has been included. In this case, drawing a horizontal line at the level of the required value of $k L$, one can get the value of $r$ that assures the desired value of overall error. Then, solving equation (18) for $n$, the number of divisions in any of the three directions is calculated as: 


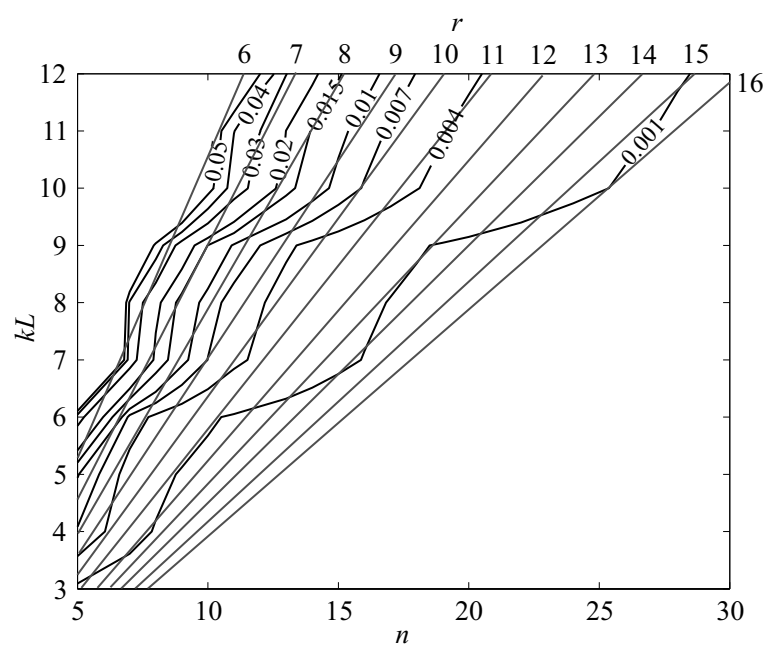

Figure 5: Number of computational cells per wavelength for given overall error and value of $k L$.

$$
n>1+r \frac{f L}{c_{0}}
$$

where the 1 is included to ensure being on the safe side, so that $n$ has to be rounded down to the nearest lower integer.

\section{Three-dimensional analysis}

In this section, first the formalism which enables to consider three-dimensional networks [2] is briefly summarized. Then, the results obtained with the proposed methodology for two simple geometries in the absence of a mean flow are compared to those obtained from FEM and full CFD computations and from experiments.

\subsection{Assembling of the three-dimensional acoustic network}

It was seen in section 3 that, in the one-dimensional case, both 2-port and multi-ports could be represented by transfer matrices, and the overall result obtained directly from the cascading property. However, in the three-dimensional case the order of the multi-ports (i.e., the number of 2-ports to which they are connected) will be in general higher than 2 , so that the transfer matrix representation is no longer suitable. Consider the multi-port represented in Figure 6, where the different wave components propagating in the adjacent 2-ports are represented. Assuming that only plane waves propagate, these wave components are related to the pressure and mass velocity fluctuations by:

$$
v_{n}^{(i)}=\frac{1}{Z_{n}^{i}}\left(p_{n}^{i+}-p_{n}^{i-}\right) ; p_{n}^{(i)}=p_{n}^{i+}+p_{n}^{i-} ; i=1, \cdots m_{n}
$$

Here, $Z_{n}^{i}=c_{0} / A_{i}$ is the characteristic impedance of the $i$-th 2-port, $A_{i}$ its cross-sectional area and $m_{n}$ is the order of the multi-port. The total mass velocity fluctuation $v_{n}$ across the multi-port may now be written as:

$$
\sum_{i=1}^{m_{n}} \frac{1}{Z_{n}^{i}}\left(p_{n}^{i+}-p_{n}^{i-}\right)=v_{n}
$$




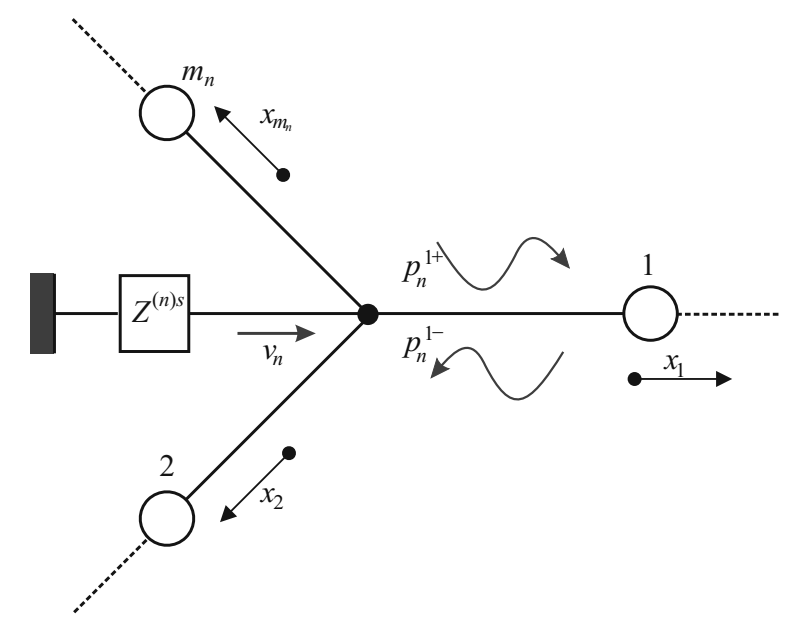

Figure 6: Acoustic representation of multi-port number $n$.

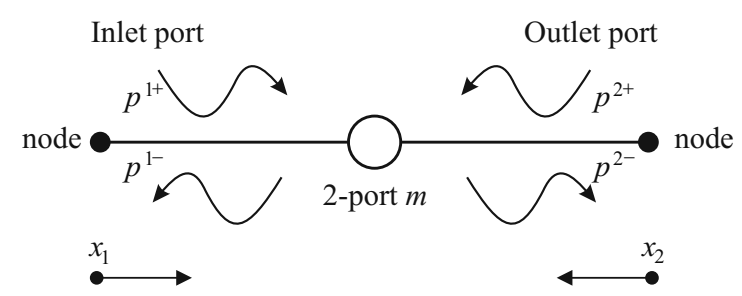

Figure 7: Scattering matrix formulation for 2-port number $m$.

Continuity of pressure across the multi-port gives:

$$
p_{n}^{i+}+p_{n}^{i-}=p_{n} \quad ; \quad i=1, \cdots m_{n}
$$

Therefore, from equations (3), (4), (21) and (22) one can write:

$$
\left[\frac{1}{Z_{n}^{1}}+\frac{1}{Z^{n(s)}}\right] p_{n}^{1+}+\sum_{k=2}^{m_{n}} \frac{1}{Z_{n}^{k}} p_{n}^{k+}=\left[\frac{1}{Z_{n}^{1}}-\frac{1}{Z^{n(s)}}\right] p_{n}^{1-}+\sum_{k=2}^{m_{n}} \frac{1}{Z_{n}^{k}} p_{n}^{k-}
$$

This can be conveniently written in matrix form as:

$$
\mathbf{S}_{n}^{+} \mathbf{p}_{n}^{+}=\mathbf{S}_{n}^{-} \mathbf{p}_{n}^{-}
$$

Here, $\mathbf{S}_{n}^{+}$and $\mathbf{S}_{n}^{-}$are the positive and negative $m_{n} \times m_{n}$ scattering matrices for the $n$-th multi-port and $\mathbf{p}_{n}^{+}$and $\mathbf{p}_{n}^{-}$are $m_{n}$-vectors of travelling pressure amplitudes moving outwards from and towards the $n$-th multi-port. In this way, a compact representation of the behaviour of the volumes regarding mass exchanges is available.

Consistently with the multi-port description provided by equation (24), the best representation for these 2 -ports is that provided by the scattering matrix, which is described in Figure 7 . Again, $p^{+}, p^{1}, p^{2+}$ and $p^{2-}$ are the travelling pressure amplitudes, whose values at each side of the 2-port are considered as state variables, which are related by means of the scattering matrix $\mathbf{S}_{m}$ of the 2-port, as:

$$
\left[\begin{array}{c}
p^{1-} \\
p^{2-}
\end{array}\right]=\mathbf{S}_{m}\left[\begin{array}{l}
p^{1+} \\
p^{2+}
\end{array}\right]
$$




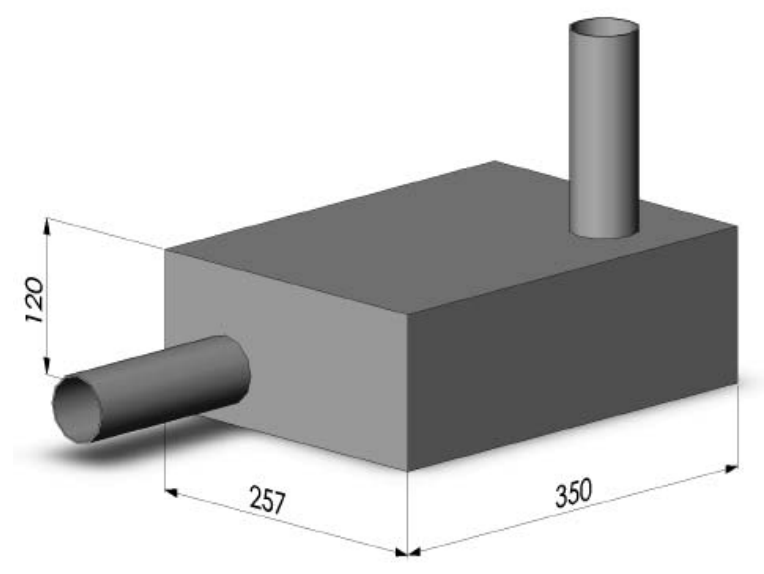

Figure 8: Rectangular expansion chamber analyzed (dimensions in $\mathrm{mm}$ ).

The elements of the scattering matrix for the momentum-related 2-ports may be readily obtained from the transfer matrix given in equation (11).

At this point, the simultaneous consideration of mass and momentum conservation has been translated into the simultaneous solution of equation (24) for each multi-port (i.e. each volume) and of equation (25) for each 2-port (i.e. each interface between volumes). Considering an acoustic network with $M$ 2-ports, equations (24) and (25) give a total of $4 M$ equations with $4 M$ unknowns, so that it is possible to combine the multi-port equations (24) and the 2-port equations (25) to derive a single equation for the whole acoustic network. This complete network equation may be written as [2]:

$$
\mathbf{A p}_{c}^{+}=\mathbf{B} \mathbf{p}_{c}^{-}
$$

Here, $\mathbf{p}_{c}^{+}$and $\mathbf{p}_{c}^{-}$are $2 M$-vectors containing the forward and backward pressure amplitudes at the endpoints of all the 2-ports in the network, and $\mathbf{A}$ and $\mathbf{B}$ denote, respectively, the left and right network matrices, which are generated by using the characteristics of the 2-ports and the multi-ports. The details of the procedure, which is based on the definition of a global incidence matrix, can be found in reference [2]. Then, if $\mathbf{p}_{c}^{+}$is known, $\mathbf{p}_{c}^{-}$can be obtained. Hence, all the travelling pressure amplitudes are known and the complete acoustic state of the network is determined. From this overall representation, it is possible to compute the transfer matrix and the Transmission Loss between any two arbitrary multi-ports.

\subsection{Validation in simple geometries}

In order to validate the methodology proposed for analyzing systems including cavities exhibiting threedimensional effects, first the acoustic response of a rectangular expansion chamber, whose dimensions are shown in Figure 8, was analyzed. The location of the inlet and outlet ducts was chosen with the purpose of exciting the maximum number of higher order modes. For the application of the proposed procedure, it was assumed that accurate results up to a frequency of $1500 \mathrm{~Hz}$ were required. The number of divisions in each direction was calculated by following the steps outlined in section 3 . Thus, in order to obtain solutions with an overall error of less than $0.7 \%$ ( $r=10$ for the prescribed frequency limit) the mesh was defined to have 12,6 and 16 divisions in the $x, y$ and $z$ directions, respectively.

As a first check, the results obtained were compared to those provided by FEM, the standard method for 3D acoustics. Two different FEM computations were considered for comparison: first, ten-node quadratic tetrahedral elements with an approximate size of $0.02 \mathrm{~m}$ (i.e. about 10 elements per wavelength at $1500 \mathrm{~Hz}$ ) 


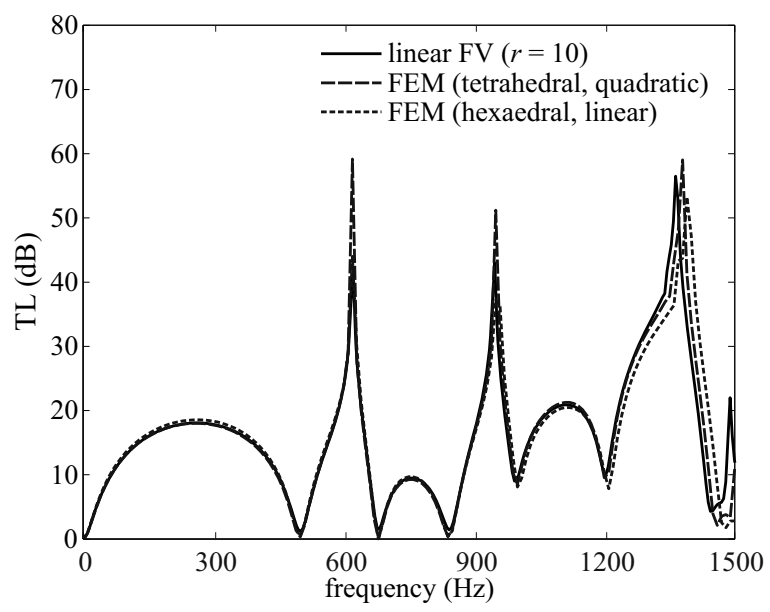

Figure 9: Transmission loss of rectangular expansion chamber: proposed methodology and FEM.

were used, so that the mesh had 10930 elements with 16678 nodes. In order to consider a case with a similar mesh size as that used in the proposed procedure, a second FEM computation was performed in which eightnode linear hexahedral elements were used, so that the mesh had 3363 elements with 2163 nodes. All three computations are plotted in Figure 9, where it is apparent that the results are qualitatively very similar. In particular, the three computations predict correctly the cut-on frequency of the first transversal mode (675.5 $\mathrm{Hz})$, while some differences may be observed around that of the second transversal mode $(1446.6 \mathrm{~Hz})$ : the proposed methodology and FEM with quadratic elements provide essentially the same value (around $1460 \mathrm{~Hz}$ ) whereas FEM with linear elements gives a value of around $1480 \mathrm{~Hz}$. Also, small differences in the frequency associated with the spike located just before this second cut-on frequency may be observed between the three computations. It is therefore apparent that the proposed procedure provides results of a quality similar to those of FEM when applied to a cavity, while still being able to handle efficiently any one-dimensional portions of the acoustic network under study.

As a second check, it seemed pertinent to compare the results of the linear finite-volume method proposed with those of a time-domain finite-volume CFD computation even if, as commented in the introduction, the use of such a method would only be reasonable if dissipative effects associated with nonlinearities or the presence of a mean flow would be expected to have any relevance, which is not the case. The methodology proposed by Broatch et al. [16] was used in this case and, similarly to the case of FEM, two CFD computations were considered: one with an optimum mesh as prescribed on the basis of a meshindependence study in [16], which would correspond to an equivalent $r$ of approximately 46, and the other one with a coarse mesh comparable to that used in the proposed procedure, i.e. with an equivalent $r$ of the order of 10. The corresponding comparison is shown in Figure 10, where a less demanding linear finite-volume computation with $r=7$ (overall error of less than $3 \%$ ) has been also included.

It can be observed that the results obtained from the proposed procedure and the CFD computation with $r \sim 46$ are rather similar, with the only exception of the cut-on frequency of the second transversal mode (which appears to be slightly overestimated by the CFD computation) and of the position of the nearby spike, similarly to what could be observed in Figure 9. However, it is apparent that the coarse-mesh CFD computation with $r \sim 10$ is not able to reproduce the expected behaviour properly, except for frequencies below the first pass-band, whereas the proposed procedure still provides reasonably good results when an even coarser discretization is used. In this sense, the proposed frequency-domain approach seem to be more efficient than the time-domain approach if a similar meshing is used. It should be remarked that the 


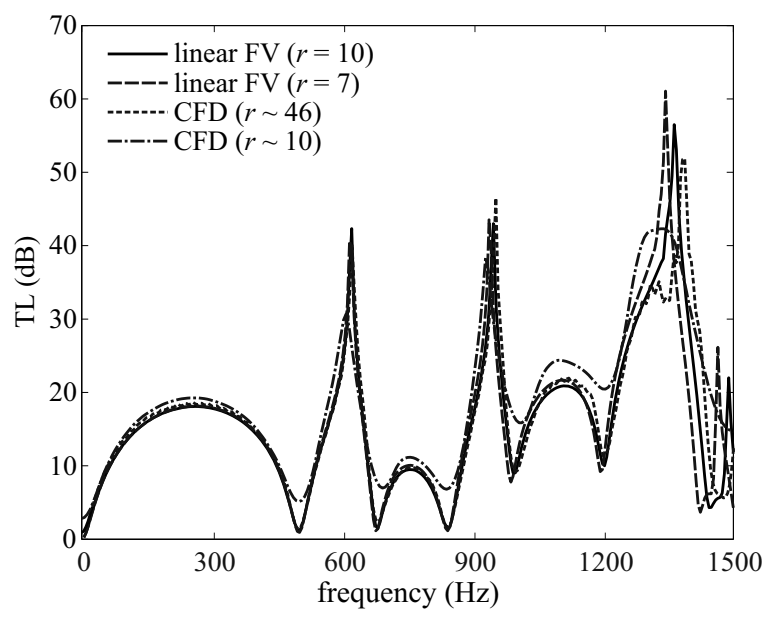

Figure 10: Transmission loss of rectangular expansion chamber: proposed methodology and CFD.

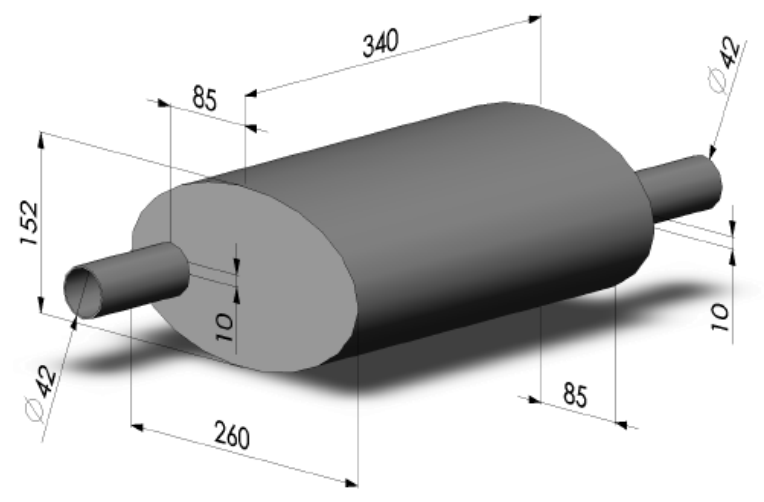

Figure 11: Elliptical expansion chamber analyzed (dimensions in $\mathrm{mm}$ ).

computation time with the methodology developed is $70 \%$ smaller than that required by the well-posed CFD computation.

In order to validate the proposed methodology in a more realistic muffler, the acoustic response of an expansion chamber with elliptical cross-section and off-set inlet and outlet was also studied. Figure 11 shows the geometry and dimensions of the expansion chamber analyzed. In this case, only experimental data has been used for validation purposes. Again, solutions with an overall error of less than $0.7 \%$ and $3 \%$ up to $1500 \mathrm{~Hz}$ were required which, according to the procedure described in section 3, imply meshes composed by 12,7 and 15 , and 9,5 and 11 , divisions in the $x, y$ and $z$ directions, respectively. In this case, comparison with experimental data obtained with a modified impulse method [28] was used in order to check the results.

Comparison between the results obtained with the proposed methodology and experimental data is shown in Figure 12. Good agreement in the location of the pass-bands is observed up to relatively high frequencies, and there are some discrepancies in the level of both attenuation peaks and pass-bands, as it could be expected, since the proposed methodology does not account for the dissipative effects associated with the excitation used [16, 28]. Again, it is observed that the proposed method gives reasonably good results even with very modest computational demands. 


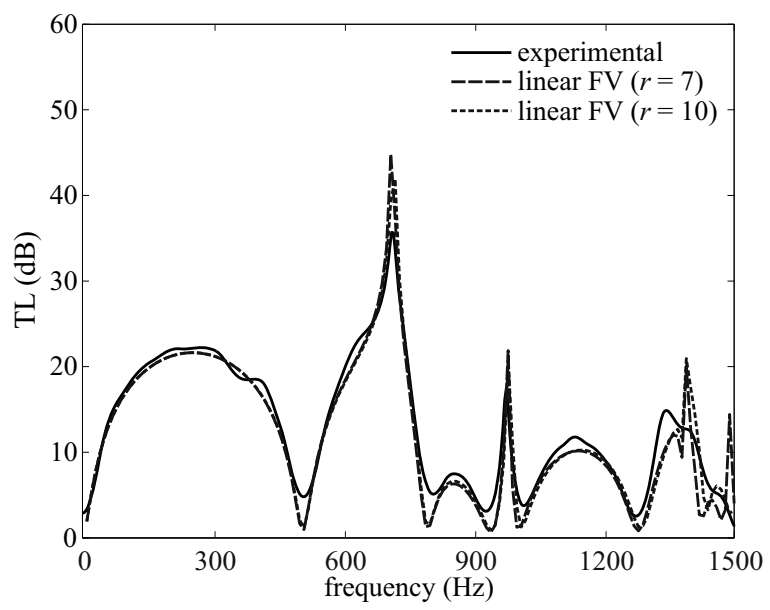

Figure 12: Transmission loss of elliptical expansion chamber: experimental measurements and proposed methodology.

\section{Conclusions}

A procedure allowing for the analysis in terms of zero- and one-dimensional elements of complex acoustic networks including cavities exhibiting three-dimensional effects has been presented and validated. The methodology is based on a linearized version of the finite volume method, which is shown to be translatable into an acoustic network comprising zero-dimensional multi-ports, which account for mass exchanges between the finite volumes, and equivalent 2-ports describing momentum exchange across the volume surfaces. The concept has been first applied to the simple one-dimensional case of a uniform duct without mean flow, where it has been possible to represent both the multi-ports and the 2-ports by means of transfer matrices. A general expression for the corresponding transfer matrix has been obtained as a function of the number of divisions used, showing that the result obtained actually converges to the exact analytical solution when the number of divisions is sufficiently large. As a by-product of this analysis, an objective criterion for the choice of a mesh providing results with a prefixed error up to a certain Helmholtz number has been obtained. This criterion has then been generalized to obtain the corresponding mesh requirements in a three-dimensional geometry. Then, a brief outline of the formalism used to solve the acoustic network representing a three-dimensional cavity has been given; in fact, in such a context the present contribution appears as a natural extension of the method proposed by Glav and Åbom [2]. The procedure has then been validated against experimental results and other numerical procedures in two simple but significant cases in the absence of a mean flow, indicating its ability to reproduce the observed three-dimensional behaviour of both rectangular and elliptical expansion chambers with a relatively moderate computational cost. Work in ongoing on the application of the proposed procedure to silencer configurations with perforated elements subject to different flow incidences, and on the consideration of the effect of absorbent materials.

\section{Acknowledgements}

This work has been partially supported by Ricardo Software, and by Ministerio de Ciencia e Innovación through grant DPI2009-14290. The authors thank Dr. F. D. Denia for his kind computational assistance.

\section{References}

[1] W. Eversman, A systematic procedure for the analysis of multiply branched acoustic transmission lines, Journal of Vibration, Acoustics, Stress and Reliability in Design - Transactions of the ASME 109 (1987) 168-177. 
[2] R. Glav, M. Åbom, A general formalism for analyzing acoustic 2-port networks, Journal of Sound and Vibration 202 (1997) 739-747.

[3] J. F. Dowling, K. S. Peat, An algorithm for the efficient acoustic analysis of silencers of any general geometry, Applied Acoustics 65 (2004) 211-227.

[4] S. N. Panigrahi, M. L. Munjal, Plane wave propagation in generalized multiply connected acoustic filters, Journal of the Acoustical Society of America 118 (2005) 2860-2868.

[5] C. Q. Howard, B. S. Cazzolato, C. H. Hansen, Exhaust stack silencer design using finite element analysis, Noise Control Engineering Journal 48 (2000) 113-120.

[6] T. Tsuji, T. Tsuchiya, Y. Kagawa, Finite element and boundary element modelling for the acoustic wave transmission in mean flow medium, Journal of Sound and Vibration 255 (2002) 849-866.

[7] A. F. Seybert, C. Y. R. Cheng, Application of the boundary element method to acoustic cavity response and muffler analysis, Journal of Vibration, Acoustics, Stress and Reliability in Design - Transactions of the ASME 109 (1987) 15-21.

[8] T. W. Wu, P. Zhang, C. Y. R. Cheng, Boundary element analysis of mufflers with an improved method for deriving the four-pole parameters, Journal of Sound and Vibration 217 (1998) 767-779.

[9] J. G. Ih, The reactive attenuation of rectangular plenum chambers, Journal of Sound and Vibration 157 (1992) 93-122.

[10] B. Venkatesham, M. Tiwari, M. L. Munjal, Transmission loss analysis of rectangular expansion chamber with arbitrary location of inlet/outlet by means of Green's functions, Journal of Sound and Vibration 323 (2009) 1032-1044.

[11] J. G. Ih, B. H. Lee, Analysis of higher order mode effects in the circular expansion chamber with mean flow, Journal of the Acoustical Society of America 77 (1985) 1377-1388.

[12] M. Åbom, Derivation of four-pole parameters including higher order mode effects for expansion chamber mufflers with extended inlet and outlet, Journal of Sound and Vibration 137 (1990) 403-418.

[13] A. Selamet, Z. L. Ji, Acoustic attenuation performance of circular expansion chambers with offset inlet/outlet: I. analytical approach, Journal of Sound and Vibration 213 (1998) 601-617.

[14] F. D. Denia, J. Albelda, F. J. Fuenmayor, A. J. Torregrosa, Acoustic behaviour of elliptical chamber mufflers, Journal of Sound and Vibration 241 (2001) 401-421.

[15] F. D. Denia, A. Selamet, F. J. Fuenmayor, R. Kirby, Acoustic attenuation performance of perforated dissipative mufflers with empty inlet/outlet extensions, Journal of Sound and Vibration 302 (2007) 1000-1017.

[16] A. Broatch, X. Margot, A. Gil, F. D. Denia, A CFD approach to the computation of the acoustic response of exhaust mufflers, Journal of Computational Acoustics 13 (2005) 301-316.

[17] Z. L. Ji, H. S. Xu, Z. X. Kang, Influence of mean flow on acoustic attenuation performance of straight-through perforated tube reactive silencers and resonators, Noise Control Engineering Journal 58 (2010) 12-17.

[18] W. Eversman, G. Ricci, A multiple degree of freedom cavity element for acoustic transmission-lines, Journal of Vibration, Acoustics, Stress and Reliability in Design - Transactions of the ASME 110 (1988) 76-83.

[19] A. J. Torregrosa, A. Broatch, R. Payri, The use of transfer matrix for the design of interferencial systems in exhaust mufflers, SAE Paper 2000-01-0728 (2000).

[20] A. Mimani, M. L. Munjal, Transverse plane wave analysis of short elliptical chamber mufflers: An analytical approach, Journal of Sound and Vibration 330 (2011) 1472-1489.

[21] S. N. Panigrahi, M. L. Munjal, A generalized scheme for analysis of multifarious commercially used mufflers, Applied Acoustics 68 (2007) 660-681.

[22] S. Amphlett, P. C. Niven, F. Payri, A. J. Torregrosa, Linear acoustic modelling using 1-d flow systems which represent complex 3-d components, SAE Paper 2011-01-1524 (2011).

[23] S. M. Sapsford, V. C. Richards, D. R. Amlee, T. Morel, M. T. Chappell, Exhaust system evaluation and design by non-linear modelling, SAE Paper 920686 (1992).

[24] T. Morel, J. Morel, D. A. Blaser, Fluid dynamic and acoustic modelling of concentric-tube resonators/silencers, SAE Paper 910072 (1991).

[25] M. L. Munjal, Acoustics of ducts and mufflers, Wiley, 1987.

[26] N. S. Dickey, A. Selamet, K. V. Tallio, Effects of numerical dissipation and dispersion on acoustic predictions from a time-domain finite difference technique for non-linear wave dynamics, Journal of Sound and Vibration 259 (2003) 193-208

[27] T. Elnady, M. Åbom, S. Allam, Modelling perforates in mufflers using two-ports, Journal of Vibration and Acoustics Transactions of the ASME 132 (2010) 061010.

[28] F. Payri, J. M. Desantes, A. Broatch, Modified impulse method for the measurement of the frequency response of acoustic filters to weakly nonlinear transient excitations, Journal of the Acoustical Society of America 107 (2000) 731-738. 